\title{
LC4 铝合金表面电泳沉积硅锆有机一无机杂化涂层的制备及性能
}

\author{
刘建华* 董琳 于美李松梅詹中伟 \\ (北京航空航天大学材料科学与工程学院 北京 100191)
}

\begin{abstract}
摘要 采用阴极电泳沉积的方法在 LC4 铝合金表面制备硅锆有机一无机杂化涂层, 并探讨了电泳沉积条件对涂层形貌、 结构以及耐蚀性的影响. 采用纳米粒度仪检测了不同硅锆杂化溶胶的 zeta 电位; 采用扫描电子显微镜(SEM)和原子力 显微镜(AFM)观察了涂层的表面微观形貌和粗䊁程度; 采用傅里叶红外光谱(FTIR)研究了涂层的化学结构; 采用电化 学方法研究了沉积电压对涂层耐蚀性能的影响, 进而探讨了电泳沉积增强杂化涂层耐蚀性的机理. 结果显示沉积体系 的 $\mathrm{pH}$ 为 1.6、沉积电压为 $5 \mathrm{~V}$ 时为最佳的沉积条件, 所获得的硅锆有机一无机杂化涂层表面均匀致密性最好, 粗粘程度 和耐蚀性都得到了明显的改善, 在 $3.5 \% \mathrm{NaCl}$ 溶液中体现出较好的耐蚀作用.
\end{abstract}

关键词 电泳沉积; 有机-无机杂化; 涂层; 沉积电压; 铝合金

\section{Preparation and Properties of Silicon-Zirconium Hybrid Coatings by Electrophoretic Deposition on LC4 Aluminum Alloy}

\author{
Liu, Jianhua* Dong, Lin Yu, Mei Li, Songmei Zhan, Zhongwei \\ (School of Materials Science and Engineering, Beihang University, Beijing 100191, China)
}

\begin{abstract}
Silicon-zirconium hybrid coatings were prepared by cathode electrophoretic deposition (EPD) method on the surface of LC4 aluminum alloy. The influence of deposition conditions on the morphology, structure and corrosion resistance of the hybrid coatings was studied. Silicon sol and zirconium sol with a molar ratio $\mathrm{Si} / \mathrm{Zr}=3: 1$ were prepared separately and then mixed. The silane solutions used here consist of GTMS (3-glycidoxypropyltrimethoxysilane) and absolute ethanol and deionized water mixed solvent, with $\mathrm{pH}$ adjusted by acetic acid. The obtained solutions were vigorously stirred at room temperature for $1 \mathrm{~h}$. On the other hand, TPOZ (zirconium $n$-propoxide) was complexed with ethyl acetoacetate and diluted with absolute ethanol up to a molar ratio ethanol/ $\mathrm{H}_{2} \mathrm{O}=3: 1$. The $\mathrm{Zr}$-solution was stirred for $1 \mathrm{~h}$ at room temperature. After then Si-solution and $\mathrm{Zr}$-solution were mixed under stirring at room temperature for $30 \mathrm{~min}$. Two-electrode system in which Plumbum and LC4 aluminum alloy as the anode and the cathode respectively was applied for the deposition of silicon-zirconium organic-inorganic hybrid coatings. The electrodeposition was mainly carried out at different cathodic potentials $(0,2,5,10,20 \mathrm{~V}$, respectively), for $10 \mathrm{~min}$. Finally the silane films were dried at room tempreture and then cured at 60 ${ }^{\circ} \mathrm{C}$ for $2 \mathrm{~h}, 90{ }^{\circ} \mathrm{C}$ for $30 \mathrm{~min}, 100{ }^{\circ} \mathrm{C}$ for $15 \mathrm{~min}$ at air atmosphere in an oven. Further heat treatment provides the thermal energy for the condensation reaction. The zeta potential of different solution was measured by nano-particle size analyzer. The morphology and roughness of the hybrid coatings were detected by using scanning electron microscopy (SEM) and atomic force microscope (AFM), respectively. The chemical structure of the silicon-zirconium hybrid coatings was investigated by using Fourier transform infrared spectroscopy (FTIR). The corrosion resistance of the hybrid coatings was studied by electrochemical impedance spectroscopy (EIS). Finally, the corrosion resistance mechanism of the coatings was discussed. The results showed that the optimum $\mathrm{pH}$ of the hybrid solution was 1.6 and the best cathode voltage was $5 \mathrm{~V}$, under which the hybrid coatings are of best performance with uniform surface, dense structure, less roughness, and excellent corrosion resistance in $3.5 \% \mathrm{NaCl}$ solution.
\end{abstract}

Keywords electrophoretic deposition; organic-inorganic hybrid; coatings; deposition potential; aluminum alloy

\section{1 引言}

硅锆有机-无机杂化涂层是一种均匀的多相涂层, 与金属基体的结合能力强, 具有很好的耐蚀性能 ${ }^{[1 \sim 3]}$. 无机相(由无机氧化物 $\mathrm{SiO}_{2}, \mathrm{ZrO}_{2}$ 组成)作为涂层的主体, 能够起到增强涂层强度、耐磨性、热稳定性等作用 ${ }^{[4 \sim 6]}$;
有机相(主要来源于有机硅氧烷上的碳链和功能基团)能 够增加涂层的柔韧性、透气性、疏水性等性能 ${ }^{[7,8]}$. 因此, 近年来这种材料一直是人们研究的重点.

传统的有机一无机杂化涂层, 溶胶凝胶杂化涂层的 涂覆方法包括喷涂、旋涂、浸涂等 ${ }^{[9,10]}$. 对于这些涂覆 方法, 单次涂覆得到的涂层薄, 耐蚀性较差, 多次涂覆 涂层厚度虽然有所增加, 但涂覆工艺复杂、涂覆过程中

\footnotetext{
*E-mail: liujh@buaa.edu.cn; Tel.: 010-82317103

Received May 14, 2012; published August 27, 2012.

Supporting information for this article is available free of charge via the Internet at http://sioc-journal.cn.
} 
影响因素较多, 涂层均匀性变差. 阴极电泳沉积技术完 全不同于传统涂覆技术, 可以在低浓度溶胶中获得厚度 显著提高的涂层, 大大增加了溶胶的稳定性, 且工艺简 单易行、可用于复杂形状, 成本低, 可靠性高, 因此该工 艺倍受关注 ${ }^{[11,12]}$.

Mandler 等 ${ }^{[13,14]}$ 最先将电泳沉积技术与金属防护相 结合, 在铝合金等导电金属基体上电泳沉积硅烷涂层和 氧化锆 $\left(\mathrm{ZrO}_{2}\right)$ 薄膜, 得到的涂层耐蚀性能明显优于传统 浸涂方法. Collinson 等 ${ }^{[15]}$ 、Woo 等 ${ }^{[16]}$ 也在导电基体上阴 极电泳沉积得到硅烷涂层, 并通过扫描电镜及接触角技 术研究了硅烷涂层的均匀性和取向性, 发现涂层表面结 构不同于传统涂覆方法, 涂层厚度可控, 界面结合力得 到显著提高. Castro 等 ${ }^{[17]}$ 利用阴极电泳沉积技术得到了 均一透明的氧化硅-氧化锆混合涂层, 涂层与金属表面 的结合力良好. 阴极电泳沉积技术利用有机一无机杂化 涂层制备过程中溶胶凝胶体系颗粒的带电特性和前驱 体物质碱致聚合的反应原理，使用金属基体做阴极，溶 胶中的带电粒子在电场的作用下向阴极迁移并沉积在 金属表面，形成致密的涂层结构. 随着这一过程的进行， 涂层不断增厚而形成湿涂膜, 直到涂层电阻大到几乎不 导电, 带电颗粒的沉积受阻, 因此电泳沉积所得涂层的 厚度大于浸涂涂层 ${ }^{[18]}$. 在电场的持续作用下, 已沉积的 涂层内部还会发生电渗现象, 造成涂层内部的水分进入 到溶液, 使涂层含水量减少至 3\% 5\%, 进一步促进涂 层的致密化.

本研究采用阴极电泳沉积技术, 使用简单的两电极 体系, 以环氧基有机硅烷 $(\gamma$-缩水甘油醚氧丙基三甲氧 基硅烷, GTMS)和锆醇盐(正丙醇锆, TPOZ)作为硅和锆 的前驱体, 确定合适的 $\mathrm{pH}$ 值, 在 LC4 铝合金基体表面 制备硅锆有机一无机杂化涂层, 并探讨不同沉积电压对 涂层表面形貌、内部结构以及耐蚀性的影响.

\section{2 结果与讨论}

\section{1 体系的 $\mathrm{pH}$ 值}

不同 $\mathrm{pH}$ 值硅锆混合溶胶的 zeta 电位变化情况如图 1 所示, 从中可以发现溶胶体系 $\mathrm{pH}$ 的变化显著影响 zeta 电位. 随着 $\mathrm{pH}$ 值的降低, 混合溶胶的 zeta 电位由负电 变为正电, 具体原因可以由反应式(1)和(2)来解释, 文 献[19]也有相同的发现. 当混合溶胶的 $\mathrm{pH}$ 低于 2.6 时, zata 电位随 $\mathrm{pH}$ 的降低明显增加; $\mathrm{pH}$ 降至 1.6 时, zata 电 位的变化幅度减小. Zeta 电位是胶体体系稳定性的重要 表征参数之一，反映了胶体颗粒的表面带电特性. Zeta 电位绝对值较高时, 颗粒带有较多的正电荷或负电荷而 相互排斥, 从而达到整个体系的稳定; 如果颗粒带有很 少的电荷即 zeta 电位绝对值较低时, 分子间引力大于乕 力而相互吸引, 使得整个体系不稳定而发生絮凝. 越正 的 zeta 电位表明胶体颗粒荷正电越多, 增加溶胶体系稳 定性的同时又有利于阴极电泳沉积. 当硅锆混合溶胶的
$\mathrm{pH}$ 值小于 1.6 时, zeta 电位的增加并不明显, 考虑到 $\mathrm{H}^{+}$ 离子的增多不利于缩聚反应的发生，故确定体系 $\mathrm{pH}$ 值 为 1.6 .

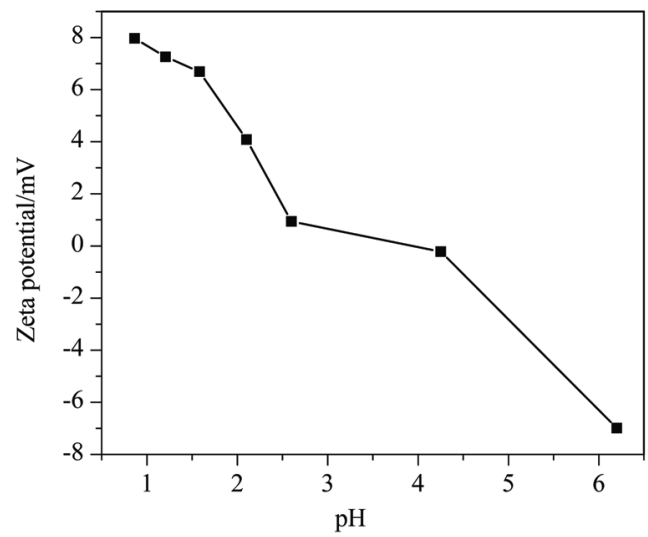

图 1 不同 $\mathrm{pH}$ 值对硅锆混合溶胶 zeta 电位的影响

Figure 1 The influences of $\mathrm{pH}$ values for the zeta potential of siliconzirconium hybrid sol

$$
\begin{aligned}
& \mathrm{M}-\mathrm{OH}+\mathrm{OH}^{-} \Leftrightarrow \mathrm{M}-\mathrm{O}^{-}+\mathrm{H}_{2} \mathrm{O} \\
& \mathrm{M}-\mathrm{OH}+\mathrm{H}^{+} \Leftrightarrow \mathrm{M}-\mathrm{OH}_{2}^{+}
\end{aligned}
$$

\section{2 涂层微观形貌及结构}

\subsection{1 杂化涂层的微观形貌}

图 2 是浸涂和电泳沉积电压为 $2,5,10,20 \mathrm{~V}$ 所制备 的硅锆有机一无机杂化涂层的扫描电镜图. 浸涂所得涂 层(图 2a)表面存在很多清晰的突起、平整度较差. 施加 $2 \mathrm{~V}$ 沉积电压获得的涂层(图 2b)表面突起明显减少, 涂 层的均一完整性大大提高. 沉积电压升高到 $5 \mathrm{~V}$ 所得的 涂层(图 2c)表面基本看不到明显的突起，涂层更加均匀， 右上角为该条件下所得涂层的局部放大图，可以看出涂 层表面均一平整. 电泳沉积电压升高到 $10 \mathrm{~V}$ (图 2d), 涂 层表面虽没有明显的突起，但已经开始出现比较明显的 孔洞，从局部放大图中可清晰地看到孔洞的存在，直径 大约为 $0.5 \mu \mathrm{m}$. 当沉积电压进一步升高到 $20 \mathrm{~V}$ 时(图 $2 \mathrm{e}$ ), 涂层表面仍比较平整, 从局部放大图中可以看到孔 洞扩展为长条状存在, 长度超过 $2 \mu \mathrm{m}$.

硅锆杂化涂层在 LC4 铝合金表面电泳沉积过程可 以用图 3 所示的阴极电泳沉积过程示意图表示. 环氧基 硅烷和正丙醇锆在水性介质中发生水解反应，形成带正 电的硅锆溶胶颗粒(步骤 A). 通电后带正电的硅锆溶胶 颗粒在电场的作用下向铝合金基体迁移(步骤 $\mathrm{B}$ ); 与此 同时，铝合金表面发生水的还原反应:

$$
\begin{aligned}
& 2 \mathrm{H}_{2} \mathrm{O}+\mathrm{O}_{2}+4 \mathrm{e}^{-} \rightarrow 4 \mathrm{OH}^{-} \\
& 2 \mathrm{H}_{2} \mathrm{O}+2 \mathrm{e}^{-} \rightarrow 2 \mathrm{OH}^{-}+\mathrm{H}_{2}
\end{aligned}
$$




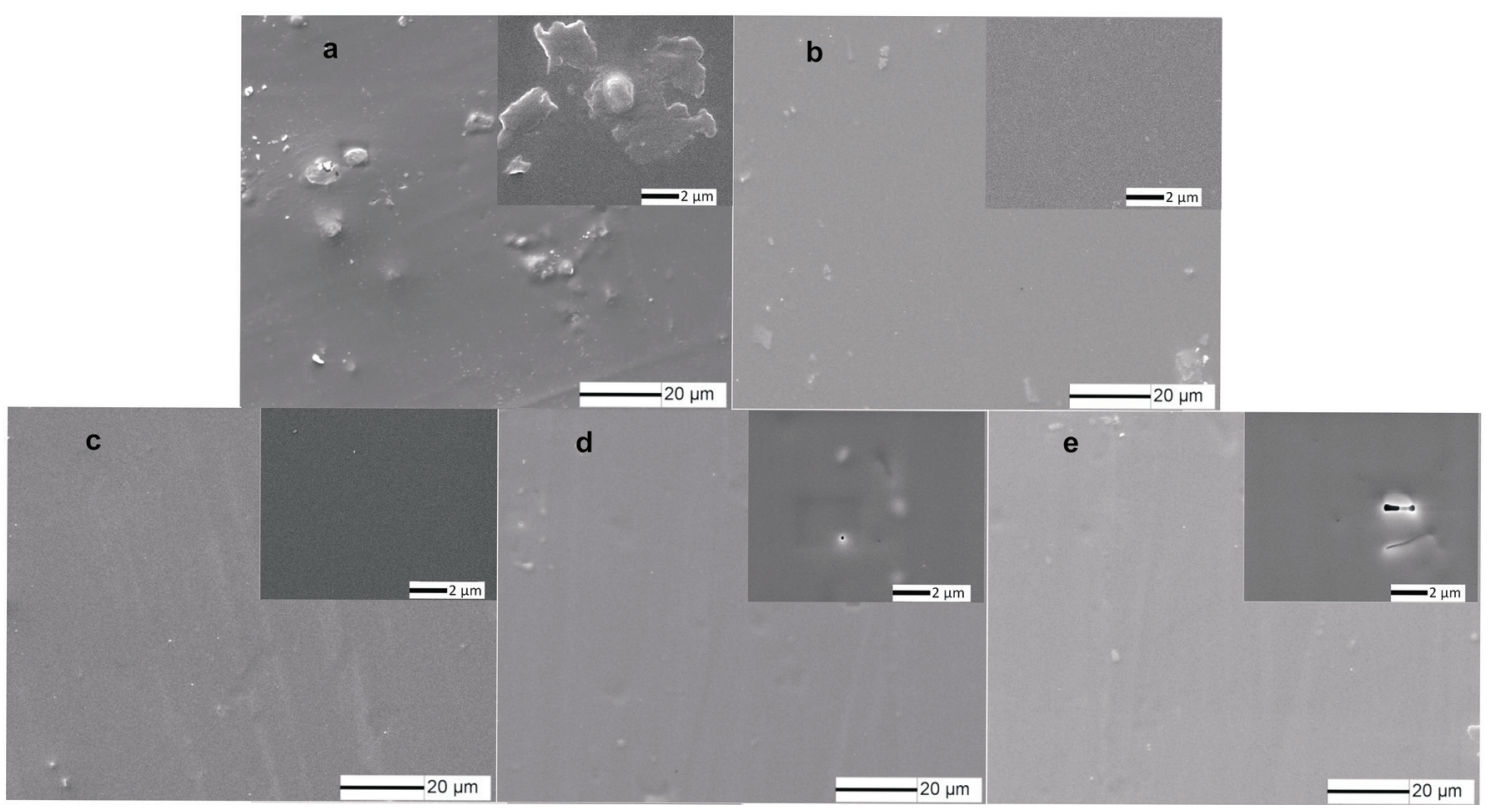

图 2 不同硅铅有机-无机杂化涂层的表面微观形貌(a 浸涂; b 电压 $2 \mathrm{~V} ; \mathrm{c}$ 电压 $5 \mathrm{~V} ; \mathrm{d}$ 电压 $10 \mathrm{~V} ; \mathrm{e}$ 电压 $20 \mathrm{~V}$ )

Figure 2 SEM images of different silicon-zirconium hybrid coatings (a: Dipping; b: $2 \mathrm{~V}$; c: $5 \mathrm{~V}$; d: $10 \mathrm{~V}$; e: $20 \mathrm{~V}$ )

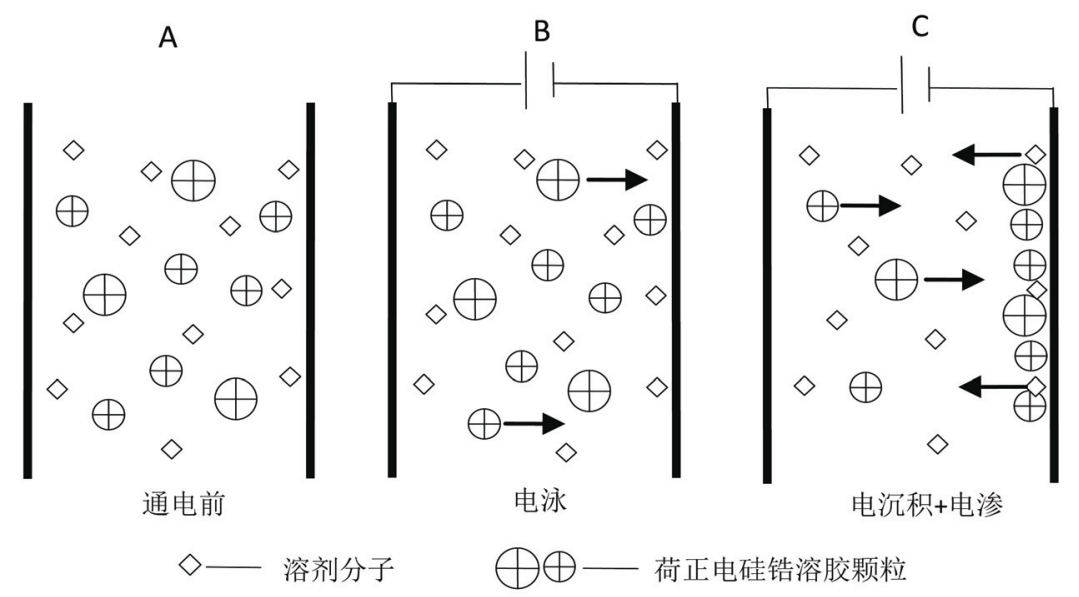

图 3 硅锆杂化溶胶阴极电泳沉积过程示意图

Figure 3 The schematic of cathodic electrophoretic deposition process of silicon-zirconium hybrid sol

生成大量的 $\mathrm{OH}^{-}$. 荷正电硅锆溶胶颗粒在电场作用下 到达铝合金表面后，铝合金基体附近生成的 $\mathrm{OH}^{-}$促进 了硅醇和锆醇缩聚反应的进行, 从而在铝合金表面形成 了致密的涂层结构, 电渗现象则进一步促进了涂层的致 密化(步骤 C).

出现图 2 所述现象是由于通电后荷正电的硅锆溶胶 颗粒在阴极金属基体表面的沉积得到促进，同时也造成 金属表面水发生还原反应(反应式 3、4)而生成了大量的 缩聚反应催化剂 $\mathrm{OH}^{-}$, 铝合金基体表面的反应活性大 大提高, 促进了硅醇和锆醇在基体表面的吸附及缩聚反 应的发生, 从而避免了浸涂过程中出现的吸附缺陷. 当 电压值进一步升高，金属基体表面由于水的还原反应而 析出的氢气不可忽视, 导致涂层表面出现了明显的孔洞 结构. 电压过大时铝合金基体可能发生阴极腐蚀, 影响 涂层的致密性和均匀性.
图 4 所示为浸涂涂层及不同电压下沉积涂层表面的 典型原子力显微镜形貌. 浸涂涂层(图 4a)表面有明显的 突起和凹坑, 粗䊁程度较大. 电压为 $2 \mathrm{~V}, 5 \mathrm{~V}$ 时沉积所 获得的涂层(图 4b，4c)表面起伏程度明显低于浸涂所得 涂层，且随着电压的升高，涂层表面的平整度、均匀性 都明显提高. 当电压达到 $10 \mathrm{~V}$ 时(图 4d), 涂层表面出现 了明显的凹坑, 这与扫描电镜的表征结果相符.

由上所述，沉积电压为 $5 \mathrm{~V}$ 时得到的涂层在铝合金 基体表面的覆盖性最好, 表面平整性高, 没有明显的孔 洞结构、涂层均匀致密.

\section{2 .2 杂化涂层的结构}

图 5 为浸涂及不同电压下沉积所得硅锆杂化涂层的 红外光谱图. 图中各个特征峰出现的位置十分相似, 其 中 $1084 \mathrm{~cm}^{-1}$ 和 $1052 \mathrm{~cm}^{-1}$ 为 GTMS 水解所得硅醇发生 
缩聚反应形成的 $\mathrm{Si}-\mathrm{O}-\mathrm{Si}$ 键的反对称伸缩振动特征 峰 ${ }^{[20,21]} .952 \mathrm{~cm}^{-1}$ 和 $1633 \mathrm{~cm}^{-1}$ 附近为硅醇与 TPOZ 水解 所得锆醇缩聚形成的 $\mathrm{Si}-\mathrm{O}-\mathrm{Zr}$ 键的振动峰 ${ }^{[22]}$. 1195 $\mathrm{cm}^{-1}$ 为未发生水解的 $\mathrm{Si}-\mathrm{O}-\mathrm{CH}_{3}$ 的振动峰. $851 \mathrm{~cm}^{-1}$ 处的特征峰是由于硅醇在铝合金基体表面的吸附及脱 水缩合反应而形成了 $\mathrm{Al}-\mathrm{O}-\mathrm{Si}$ 化学键 ${ }^{[23]} .1275 \mathrm{~cm}^{-1}$ 为环氧基的振动峰 ${ }^{[24]}$, 沉积电压为 $5 \mathrm{~V}$ 时该波数段的吸 收峰强度最大(图 5c), 说明 GTMS 沉积在铝合金表面而 没有发生开环反应，电压为 $5 \mathrm{~V}$ 时最有利于环氧基硅烷 在铝合金基体表面的沉积, 环氧基的存在能够增加涂层 的柔韧性、疏水性, 进而影响涂层的耐蚀性. 此外, 在 $3372 \mathrm{~cm}^{-1}$ 和 $980 \mathrm{~cm}^{-1}$ 附近都没有明显的吸收峰, 说明 混合溶胶体系中水解形成的 $\mathrm{Si}-\mathrm{OH}$ 键全部发生缩聚反 应或与铝合金基体表面发生反应 ${ }^{[25]}$. 上述结果表明，浸 涂和阴极电泳沉积方法都可以得到结构稳定的硅锆有 机一无机杂化涂层, 施加 $5 \mathrm{~V}$ 的沉积电压最有利于荷正 电的环氧基硅烷胶体颗粒在铝合金基体表面的覆盖沉 积, 使涂层耐蚀性更佳.

\section{3 电化学性能}

不同涂覆条件下获得的杂化涂层在 $3.5 \% \mathrm{NaCl}$ 溶液 中浸泡 $1 \mathrm{~h} 、 72 \mathrm{~h}$ 和 $120 \mathrm{~h}$ 后的电化学阻抗谱分别如图 $6 \sim 8$ 所示.
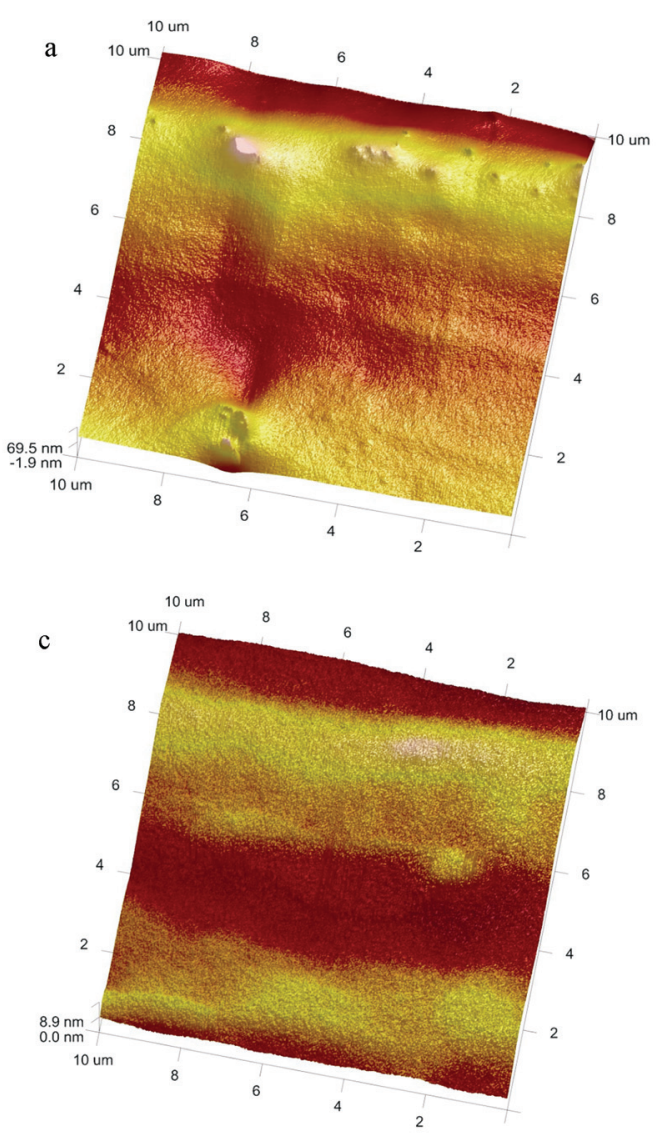

由图 6 可知, 在 $3.5 \% \mathrm{NaCl}$ 溶液中浸泡 $1 \mathrm{~h}$ 后，不同 硅锆有机一无机杂化涂层的 Bode 阻抗曲线具有相同的 变化趋势, 在测试频率范围内阻抗值随频率的降低而上 升, 说明溶胶凝胶涂层在浸泡初期很好地阻挡了水、氧 和其他离子等腐蚀物质渗透到铝合金基体，有效防止了 腐蚀的发生. 在高频段和低频段, 电泳沉积所得涂层的 阻抗值明显大于浸涂所得涂层, 这是由于沉积涂层的致 密性优于浸涂涂层，对腐蚀介质的阻挡作用比较明显. 随着沉积电压升高到 $5 \mathrm{~V}$, 低频段阻抗值比浸涂涂层提 高了一个数量级，这是由于反应粒子在基体表面的沉积 量增加, 反应程度增大, 涂层结构完整、致密性增加. 当 电压超过 $10 \mathrm{~V}$ 涂层出现孔洞结构而导致其致密性下降, 抵挡腐蚀介质的能力减弱，阻抗值也有所降低. 浸泡初 期浸涂涂层与电泳沉积涂层的 Bode 相位角图谱相似, 在低频段出现一个峰, 高频段出现一个相对较小的峰, 因此检测到两个时间常数. 高频段的时间常数是由于杂 化防护涂层的形成，低频段的时间常数则是由于前躯体 水解所形成的硅醇 $\mathrm{Si}-\mathrm{OH}$ 和锆醇 $\mathrm{Zr}-\mathrm{OH}$ 与金属基体 表面的活性基团 $\mathrm{Al}-\mathrm{OH}$ 发生脱水缩聚反应而形成共价 键，结构均匀致密. 这两个时间常数将在后续的等效电 路中有所体现.
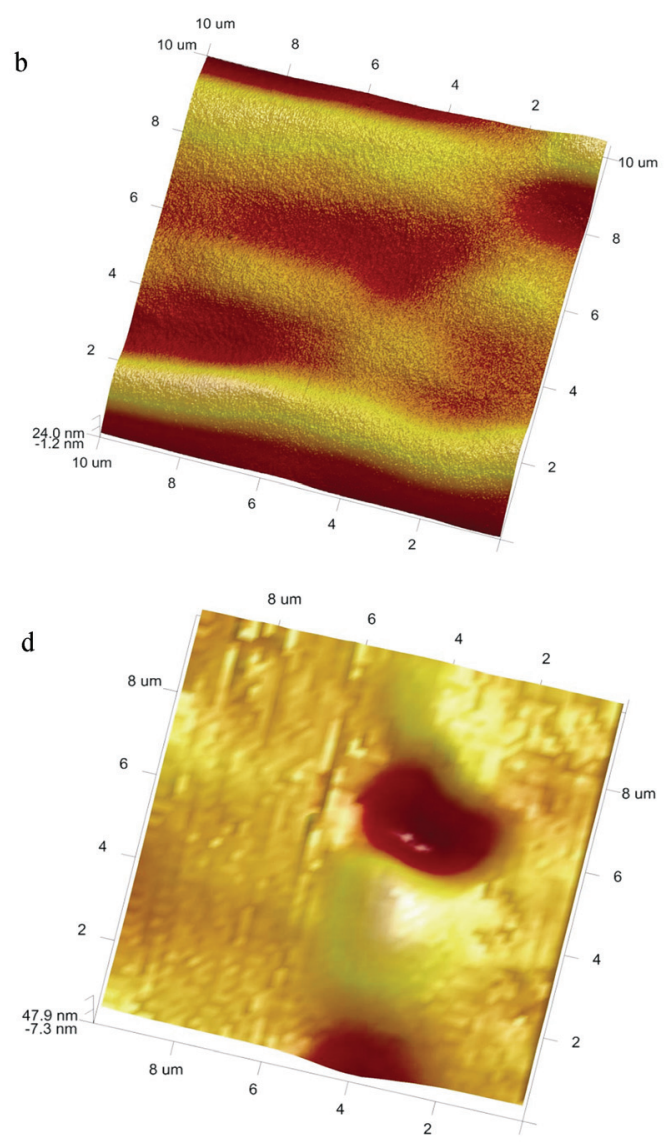

图 4 不同硅锆有机一无机杂化涂层的表面形态( $\mathrm{a}$ 浸涂; b 电压 $2 \mathrm{~V}$; c 电压 $5 \mathrm{~V} ; \mathrm{d}$ 电压 $10 \mathrm{~V}$ )

Figure 4 AFM images of different silicon-zirconium hybrid coatings (a: Dipping; b: 2 V; c: 5 V; d: 10 V) 


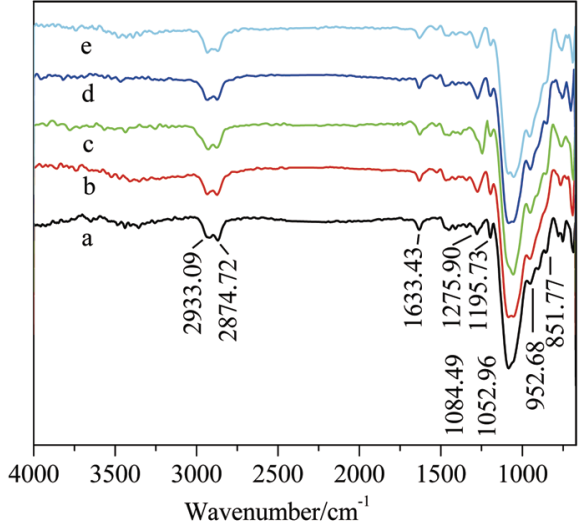

图 5 不同硅锆有机-无机杂化涂层的红外光谱图: (a)浸涂; (b) $2 \mathrm{~V}$; (c) $5 \mathrm{~V}$; (d) $10 \mathrm{~V}$; (e) $20 \mathrm{~V}$

Figure 5 FTIR spectra of different silicon-zirconium hybrid coatings (a: Dipping; b: $2 \mathrm{~V}$; c: $5 \mathrm{~V}$; d: $10 \mathrm{~V}$; e: $20 \mathrm{~V}$ )
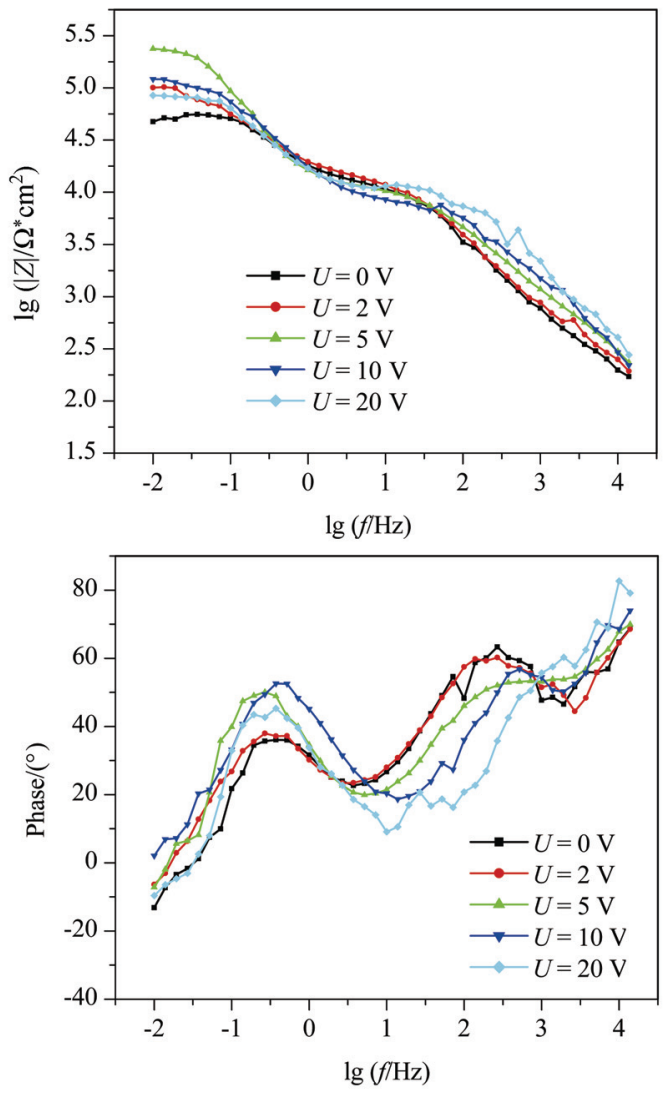

图 6 不同硅锆有机-无机杂化涂层在 $3.5 \% \mathrm{NaCl}$ 溶液中浸泡 $1 \mathrm{~h}$ 后的 电化学阻抗谱图

Figure 6 EIS of different silicon-zirconium hybrid coatings immersed in $3.5 \% \mathrm{NaCl}$ solution for $1 \mathrm{~h}$

在 3.5\% NaCl 溶液中浸泡 $72 \mathrm{~h}$ 后(如图 7), 浸涂所 得涂层在所有频率范围内的阻抗值变化明显, 尤其是低 频段大约降低了一个数量级. 而电泳沉积所得涂层的阻 抗值在测试频率范围内略有下降, 变化并不明显, $5 \mathrm{~V}$ 电压沉积所得涂层的阻抗值仍然最大. 不同杂化涂层的 Bode 相位角图谱仍具有相同的变化趋势, 中低频段的 峰变清晰变大, 高频段的平台此时已经完全消失, 中高
频段相位角降低到 $10^{\circ}$, 这些变化表明腐蚀介质(如水、 离子等)已经渗透了涂层, 并到达金属基体.
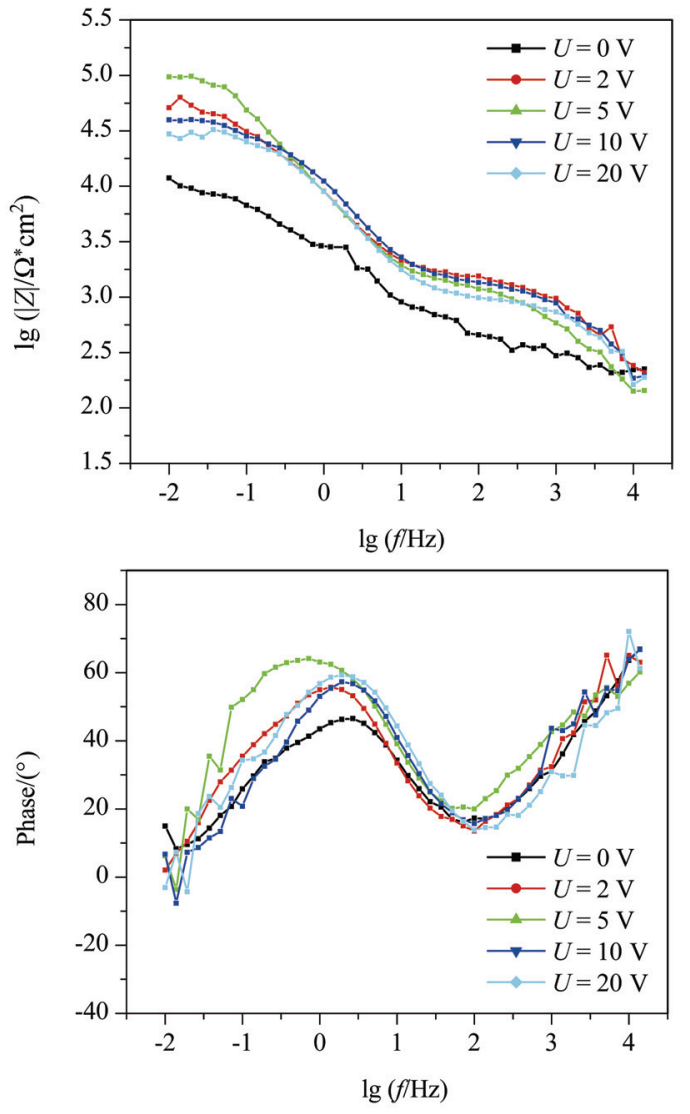

图 7 不同硅锆有机一无机杂化涂层在 $3.5 \% \mathrm{NaCl}$ 溶液中浸泡 $72 \mathrm{~h}$ 的 电化学阻抗谱图

Figure 7 EIS of different silicon-zirconium hybrid coatings immersed in $3.5 \% \mathrm{NaCl}$ solution for $72 \mathrm{~h}$

在 3.5\% NaCl 溶液中浸泡 $120 \mathrm{~h}$ (如图 8)后, 不同硅 锆有机-无机杂化涂层的阻抗值差别很明显. 与浸泡初 期相比, 浸涂涂层阻抗值降低幅度最大, 超过了两个数 量级; 沉积电压 $10 \mathrm{~V}, 20 \mathrm{~V}$ 所得涂层阻抗值降低也比较 明显, 接近一个数量级; 沉积电压为 $5 \mathrm{~V}$ 时所得涂层的 阻抗值变化较小. 从 Bode 相位角图谱中能够看出涂层 都只出现一个明显的峰，即含有一个时间常数. 浸涂涂 层与电压为 $10 \mathrm{~V}, 20 \mathrm{~V}$ 时沉积涂层的谱线形状类似，峰 出现在中频段，说明腐蚀介质已经大量渗透，此时的腐 蚀机理发生变化.

通过上述分析可以得出，电泳沉积获得的涂层与浸 涂涂层的腐蚀机制相同，而两种涂层耐蚀性的差别主要 是由于前者得到的涂层在金属表面的覆盖性、致密性大 大提高. 在此基础上, 采用等效电路对电压 $5 \mathrm{~V}$ 沉积所 得涂层浸泡 $1 \mathrm{~h}$ (浸泡初期)和电压 $20 \mathrm{~V}$ 沉积所得涂层浸 泡 $120 \mathrm{~h}$ (浸泡后期)的 EIS 数据进行拟合. 由于有机涂层 内部较复杂的反应在很多情况下会破坏涂层的完整性, 为了获得更好的拟合结果, 涂层电容 $C_{\mathrm{c}}$ 用常相位元件 $Q_{\text {c 代替. }}$ 

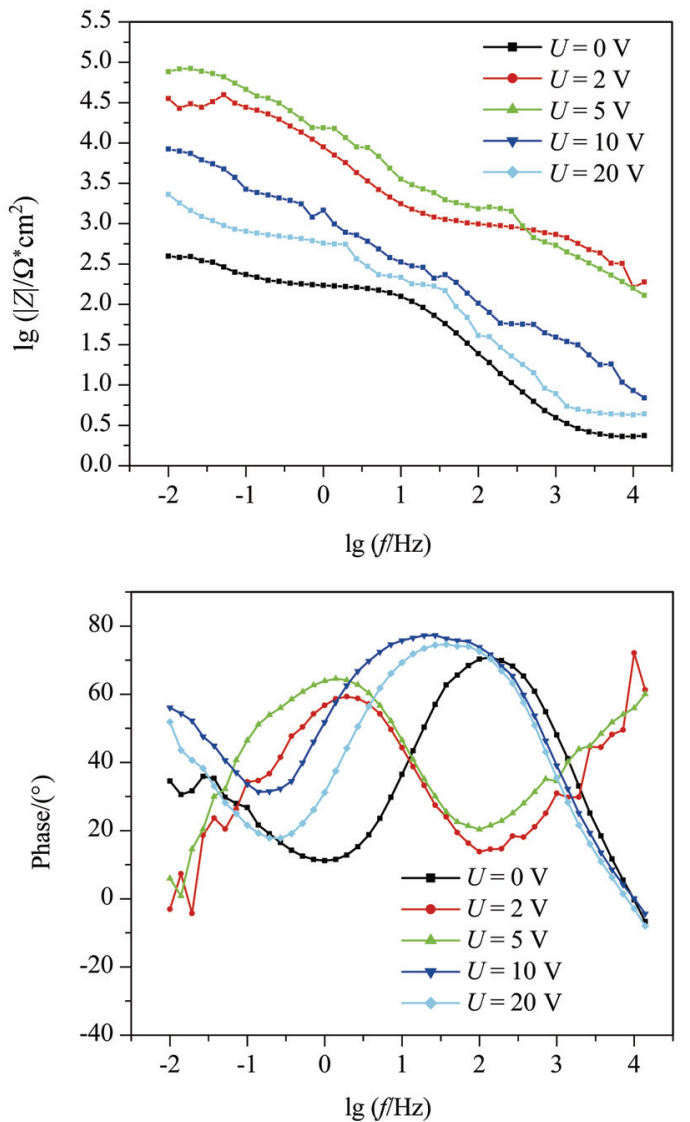

图 8 不同硅锆有机一无机杂化涂层在 $3.5 \% \mathrm{NaCl}$ 溶液中浸泡 $120 \mathrm{~h}$ 的 电化学阻抗谱图

Figure 8 EIS of different silicon-zirconium hybrid coatings immersed in $3.5 \% \mathrm{NaCl}$ solution for $120 \mathrm{~h}$

图 9 为施加电压 $5 \mathrm{~V}$ 沉积所得涂层在浸泡初期 $(1 \mathrm{~h})$ 的等效电路及拟合结果. 按照图 $9 \mathrm{a}$ 的等效电路模拟, $R_{\mathrm{el}}$ 为溶液电阻, $Q_{\mathrm{c}}$ 为涂层电容, $R_{\mathrm{p}}$ 为杂化涂层的孔洞电阻, $C_{\mathrm{o}}, R_{\mathrm{o}}$ 分别为界面氧化层的电容和电阻. 采用这种等效 电路, EIS 数据能够得到较完美的拟合. 根据拟合结果, 提取出浸泡初期不同涂层的特征参数, 列于表 1 . 电泳 沉积涂层的孔洞电阻 $R_{\mathrm{p}}$ 、界面氧化层电阻 $R_{\mathrm{o}}$ 均高于浸 涂涂层的电阻 $R_{\mathrm{p}}$ 和 $R_{\mathrm{o}}$, 且随着电压的升高而增大, 当电 压超过 $10 \mathrm{~V}$ 后, 界面氧化层电阻又开始减小, 这也是由 于电压的过度升高造成了涂层界面处的缺陷、致密性降 低, 对腐蚀介质的阻挡作用也相应地受到限制.

随着浸泡时间延长到 $120 \mathrm{~h}$, 大量的腐蚀介质和水 渗透到金属基体表面，杂化涂层和基体之间的剥离逐渐 严重, 涂层的防护性能遭到破坏, 在涂层的破坏处产生 微小的介质传输通道. Nyquist 图谱中低频弧变为斜线, 这表明扩散过程在腐蚀过程中占据了主导地位, 腐蚀机 理发生改变. 因此, 等效电路中需要增加一个反映扩散 过程的 Warburg 阻抗 $W$. 采用图 10a 的等效电路对电压 为 $20 \mathrm{~V}$ 时沉积所得涂层浸泡 $120 \mathrm{~h}$ 的 EIS 数据进行拟合, 拟合结果如图 $10 \mathrm{~b}$. 提取此阶段的主要电化学参数, 列 于表 2. 能够看到, 浸涂涂层和电压为 $10 \mathrm{~V}, 20 \mathrm{~V}$ 时电泳
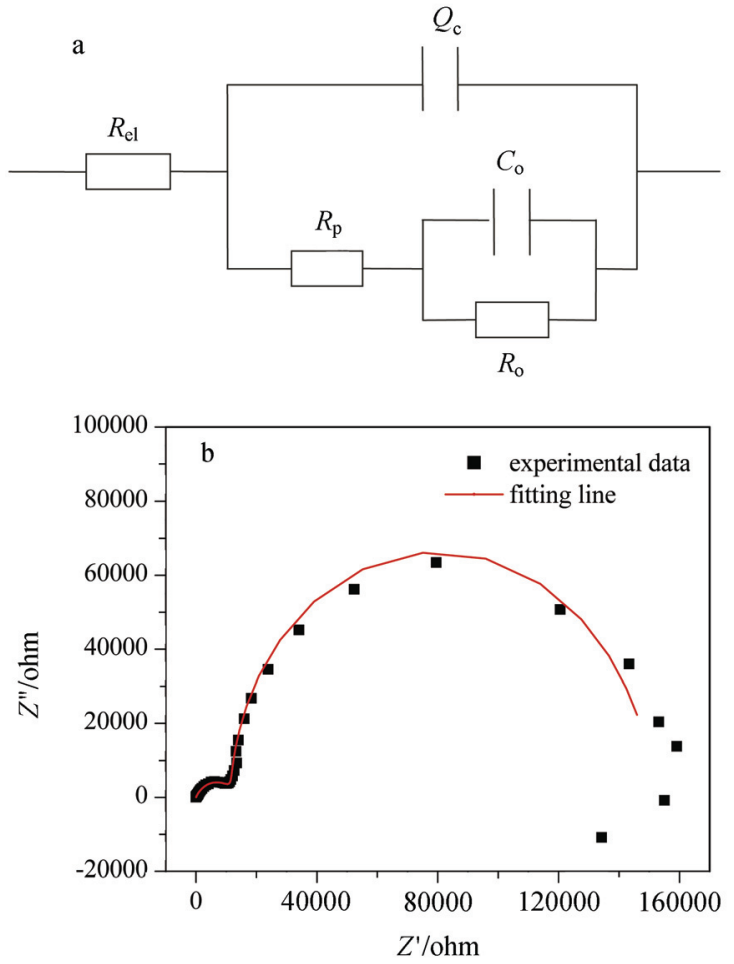

图9 $5 \mathrm{~V}$ 电压沉积所得涂层在浸泡初期的等效电路(a)及 Nyquist 拟合 结果(b)

Figure 9 Equivalent circuit (a) and Nyquist fitting result of EPD coating (5 V) (b) during early immersion time

表 1 不同杂化涂层在浸泡初期的电化学参数

Table 1 EIS parameters of different hybrid coatings during early immersion time in $3.5 \% \mathrm{NaCl}$ solution

\begin{tabular}{ccc}
\hline 沉积电压 $/ \mathrm{V}$ & $\begin{array}{c}\text { 涂层孔洞电阻 } \\
R_{\mathrm{p}} /\left(\Omega \cdot \mathrm{cm}^{2}\right)\end{array}$ & $\begin{array}{c}\text { 界面氧化层电阻 } \\
R_{\mathrm{o}} /\left(\Omega \cdot \mathrm{cm}^{2}\right)\end{array}$ \\
\hline 浸涂 & 9336 & 41400 \\
2 & 13510 & 55920 \\
5 & 19730 & 115900 \\
10 & 11220 & 87380 \\
20 & 11680 & 66750 \\
\hline
\end{tabular}

沉积涂层的 $R_{\mathrm{p}}$ 和 $R_{\mathrm{o}}$ 值都急剧减小, 表明这些涂层的腐 蚀非常严重，其保护作用已经很微弱，此时的防护作用 主要来自于涂层自身的封闭效应，而这是无法完好保护 基体免受腐蚀的.

综上所述，对于本文所研究的硅锆有机 - 无机杂化 涂层体系, 施加 $5 \mathrm{~V}$ 的沉积电压所获得的涂层表面均匀 致密、结构完整、耐蚀性能最佳.

\section{3 结论}

(1)采用阴极电泳沉积方法在 LC4 铝合金基体表面 成功制备出硅锆有机-无机杂化涂层.

(2)硅铅溶胶颗粒表面所带电荷及电荷数受硅锆混 合溶胶的 $\mathrm{pH}$ 值影响较大. $\mathrm{pH}$ 值为 1.6 时最有利于颗粒 在基体表面的沉积及反应. 

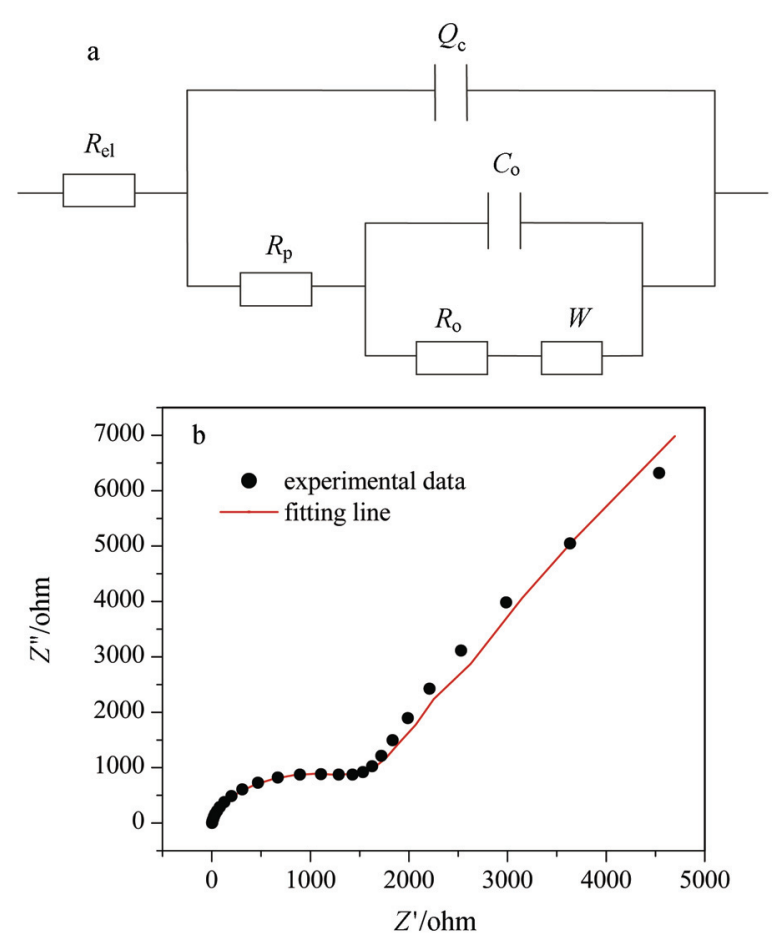

图 $1020 \mathrm{~V}$ 电压沉积所得涂层在浸泡后期的等效电路(a)及 Nyquist 拟合结果(b)

Figure 10 Equivalent circuit (a) and Nyquist fitting result of EPD coating $(20 \mathrm{~V})(\mathrm{b})$ during late immersion time

表 2 不同杂化涂层浸泡 $120 \mathrm{~h}$ 后的电化学参数

Table 2 EIS parameters of different hybrid coatings immersing in 3.5\% $\mathrm{NaCl}$ solution after $120 \mathrm{~h}$

\begin{tabular}{ccc}
\hline 沉积电压 $/ \mathrm{V}$ & 涂层孔洞电阻 $R_{\mathrm{p}} /\left(\Omega \cdot \mathrm{cm}^{2}\right)$ & 界面氧化层电阻 $R_{\mathrm{o}} /\left(\Omega \cdot \mathrm{cm}^{2}\right)$ \\
\hline 浸涂 & 15.25 & 540.4 \\
2 & 9174 & 33590 \\
5 & 13570 & 82010 \\
10 & 1822 & 3122 \\
20 & 665.8 & 1637 \\
\hline
\end{tabular}

(3)当沉积电压为 $5 \mathrm{~V}$ 时, 所获得的硅锆有机 -无机 杂化涂层在 LC4 铝合金表面覆盖性良好，涂层表面均匀 平整, 结构完整致密, 耐蚀性最佳, 可以在较长时间内 有效地阻挡腐蚀介质渗透到金属基体.

(4)电泳沉积方法和浸涂方法所得涂层的耐蚀机制 相同，耐蚀性的差别主要在于前者所得涂层在铝合金基 体表面的覆盖性以及涂层的致密性大大提高.

\section{4 实验部分}

\section{1 硅锆有机一无机杂化涂层的制备}

硅溶胶的制备, 将 GTMS 加入无水乙醇和去离子水 的混合溶剂中(醇水体积比 $3: 1$ ), 封闭搅拌 $1 \mathrm{~h}$, 使硅烷 充分水解. 锆溶胶使用乙酰乙酸乙酯为催化剂, 首先将 其与无水乙醇混合均匀, 再缓慢加入 TPOZ, 封闭搅拌 1 h. 将锆溶胶缓慢加入到硅溶胶中, 得到硅锆混合溶胶, 用硝酸调节体系 $\mathrm{pH}(1 \sim 6)$, 室温反应 $1 \mathrm{~h}$ 待用, 保证总 体系中 GTMS 与混合溶剂的体积比为 $2: 5$, 硅锆物质 的量比为 $3: 1$.

杂化涂层的阴极电泳沉积在 $25{ }^{\circ} \mathrm{C}$ 下, 采用铝合金 基体为阴极, 铅板为阳极, 分别在 $0 \mathrm{~V}, 2 \mathrm{~V}, 5 \mathrm{~V}, 10 \mathrm{~V}$ 和 $20 \mathrm{~V}$ 的电压下沉积 $10 \mathrm{~min}$, 其中施加 $0 \mathrm{~V}$ 电压所获得的 即为浸涂涂层. 涂覆后的试样首先置于空气中干燥 10 $\min$, 然后放在烘箱中 $60{ }^{\circ} \mathrm{C}$ 干燥 $2 \mathrm{~h}, 100{ }^{\circ} \mathrm{C}$ 干燥 $0.5 \mathrm{~h}$.

\section{2 测试方法}

采用纳米粒度仪检测体系中粒子颗粒尺寸及 zeta 电位. 采用傅里叶变换红外线光谱分析仪检测铝合金基 体表面所制备涂层的基团信息. 采用扫描电子显微镜对 铝合金上的溶胶凝胶涂层表面微观形貌进行观察. 采用 原子力显微镜进一步观察涂层表面形貌，并检测涂层的 表面粗糙程度. 采用电化学工作站检测杂化涂层在 $3.5 \% \mathrm{NaCl}$ 溶液中的电化学性能. EIS 测试结果采用 ZSimpwin 软件进行拟合分析.

\section{References}

[1] Wang, D. H.; Gordon, P. B. Prog. Org. Coat. 2009, 64, 37.

[2] Feng, Z.; Liu, Y.; Thompson, G. E.; Skeldon, P. Electrochim. Acta 2010, 55, 3518 .

[3] Li, Q.; Gu, M.; Du, Y. G.; Xian, X. D. Acta Chim. Sinica 2012, 70, 572. (李强，古敏，杜云贵，鲜晓东，化学学报, 2012, 70, 572.)

[4] Gupta, G.; Pathak, S. S.; Khanna, A. S. Prog. Org. Coat. 2012, 74, 106.

[5] Huang, Z. Y.; Zhang, L.; Liang, G. C. Acta Chim. Sinica 2012, 70, 235. (黄紫洋, 张岚, 梁广超, 化学学报, 2012, 70, 235.)

[6] Liu, J. H.; Wang, Q.; Yu, M.; Zhan, Z. W.; Li, S. M.; Zhan, J.; Wang, M. Chinese J. Inorg. Chem. 2012, 28, 873. (刘建华, 王强, 于美, 詹 中伟, 李松梅, 詹俊, 王邈, 无机化学学报, 2012, 28, 873.)

[7] Zhang, J. X.; Zhang, X. P.; Sun, X. T.; Liu, Y. L. Chinese J. Inorg. Chem. 2011, 27, 264. (张静媡, 张新平, 孙学通, 刘应亮, 无机化 学学报, 2011, 27, 264.)

[8] Zheng, S. X.; Li, J. H. J. Sol-Gel Sci. Technol. 2010, 54, 174.

[9] Noriko, Y.; Hiromasa, S.; Yuji, K.; Shingo, K. J. Mater. Sci. 2002, 37, 2071.

[10] Croes, K. J.; Vreugdenhil, A. J.; Maocheng, Y.; Singleton, T. A.; Boraas, S.; Gelling, V. J. Electrochim. Acta 2011, 56, 7796.

[11] Van Ooij, W. J.; Zhua, D.; Stacya, M.; Setha, A.; Mugadaa, T. J.; Gandhia, P. P. Tsinghua Sci. Technol. 2005, 10, 639.

[12] Hu, J. M.; Liu, L.; Zhang, J. Q.; Cao, C. N. Electrochim. Acta 2006, $51,3944$.

[13] Sheffer, M.; Groysman, A.; Mandler, D. Corros. Sci. 2003, 45, 2893.

[14] Shacham, R.; Mandler, D.; Avnir, D. Chem.-Eur. J. 2004, 10, 1936.

[15] Deepa, P. N.; Kanungo, M.; Claycomb, G.; Sherwood, P. M. A.; Collinson, M. M. Anal. Chem. 2003, 75, 5399.

[16] Woo, H.; Reucroft, P. J.; Jacob, R. J. J. Adhes. Sci. Technol. 1993, 7, 681.

[17] Castro, Y.; Ferrari, B.; Moreno, R.; Dura'n, A. J. Sol-Gel Sci. Technol. 2005, 35, 51 .

[18] Zhitomirsky, I. Adv. Colloid Interface 2002, 97, 279.

[19] Kim, J.; Wong, P. C.; Wong, K. C.; Sodhi, R. N. S.; Mitchell, K. A. R. Appl. Surf. Sci. 2007, 253, 3133. 
[20] Susac, D.; Sun, X.; Mitchell, K. A. R. Appl. Surf. Sci. 2003, 207, 40.

[21] Pathak, S. S.; Sharma, A.; Khanna, A. S. Prog. Org. Coat. 2009, 65, 206.

[22] Castro, Y.; Aparicio, M.; Moreno, R.; Dura'n, A. J. Sol-Gel Sci. Technol. 2005, 35, 41.

[23] Hu, J. M.; Liu, L.; Zhang, J. Q.; Cao, C. N. Prog. Org. Coat. 2007,
$58,265$.

[24] Chen, M. A.; Xie, X.; Zhang, X. M. Prog. Org. Coat. 2009, 66, 40.

[25] Wu, K. H.; Chao, C. M.; Yeh, T. F.; Chang, T. C. Surf. Coat. Technol. 2007, 201, 5782 .

(Cheng, B.; Lu, Z.) 